\title{
Consumer Demographics and Self-Medication: A Cross-Sectional Study of Karachi City in Pakistan
}

\author{
Sheikh M. Fakhre Alam siddiqui ${ }^{1 *}$ \\ Dr.Shameel A. Zuberi ${ }^{2}$ \\ Sohaib Uz Zaman ${ }^{3}$ \\ 1. Karachi University Business School, University of Karachi. Main University Road Karachi - 75270. \\ Sindh, Pakistan . \\ 2. Karachi University Business School, University of Karachi. Main University Road Karachi - 75270. \\ Sindh, Pakistan. \\ 3. Karachi University Business School, University of Karachi. Main University Road Karachi - 75270. \\ Sindh, Pakistan.
}

\begin{abstract}
The purpose of this study is to find the statistical relationship between self-medication and demographic characteristics. A cross-sectional study was conducted in the local settings of Karachi. A total of 210 individuals participated in the study. The data was collected through self-administered questionnaire. SPSS. 20 was used for data analysis. This software help in data management from above mentioned sources, and statistical analysis of the data. Chi-square and Phi and Cramer's V test was used to measure attitude of consumer towards self medications. The study shows that $82 \%$ of the surveyed people practice self-medication, mostly for minor illnesses. The common reasons for self-medication as stated by the respondents are prior experience, convenience, and recommendation. The majority of the respondent regarded self-medication as an acceptable practice and a shortterm cure to the illness. Finally, there is no statistical relationship between self-medication and demographic characteristics of the population. The sample size that has been taken is small. Thus it is possible that the results do not give the exact image of the population. Moreover, responses of the participants could be inaccurate, as participants may not have been able to correctly recall past events.
\end{abstract}

Key Words: Self-medication, Over the Counter medicines (OTC), Minor illness, Prior experience, Convenience.

DOI: $10.7176 / \mathrm{EJBM} / 11-14-04$

Publication date:May $31^{\text {st }} 2019$

\section{Introduction}

Globally, self-medication practices have been reported as being on the rise. Self-medication is defined as acquisition and use of medicines to treat one's self without consulting a licensed practitioner or doctor (WHO, 2000). Nowadays a number of households practice self-medication for minor illness (common cold, headache, flu, etc.) before consulting a doctor. And this, as a result, has increased the sales of OTC and non-OTC medicines.

The patterns of self-medication are an important indicator of health. These patterns help identify what diseases are prevalent in the population and what remedial actions are taken by the people (Dukes, 1993). Thus self-medication is an important issue as it may delay the diagnosis of disease and may give rise to more resistant pathogens (Wajngarten, 2001).

Unfortunately, self-medication practices are more common in developing countries than in developed countries. This trend can be attributed to the lack of or delay in medical facilities and expensive medical care in developing countries (Chang \& Trivedi, 2003). Moreover, in developing countries, almost all drug stores or pharmacies hand out medicines without a prescription from the practitioner (Chang \& Trivedi, 2003). So all these factors encourage self-medication practices in developing countries.

Finally, the recent development of pharmaceuticals in Pakistan has contributed to a widespread availability of medicines which in turn promotes self-medication. The most commonly available medicines are painkiller, cough and cold remedies, anti-allergy medicines, vitamins and energy tonics (Hussain \& Khanum, 2008). Although some of these medicines are risk-free and useful for the treatment of small health problems, their excessive use can cause serious side effects or adverse reactions. Also, the lack of education and awareness related to medicines makes self-medication practice more dangerous.

Despite this, there is a paucity of literature on the subject of self-medication in developing nations. So the aim of this study is to determine the relationship of self-medication and demographic characteristics of a population.

\subsection{Problem Statement}

Self-medication is one of the most critical healthcare issues which could potentially endanger a person's life. Numerous studies have been conducted over the period on this subject to identify the factors that encourage self-medication and the degree of its prevalence. These studies have identified a wide range of factors that induce/prompt people to engage in self-medication. According to a study conducted in Nepal, 59\% of 142 
respondents had practiced self-medication in the past six months, and minor illness, prior experience with the same illness \& lack of healthcare were the common reasons behind it (Shankar, Partha \& Shenoy, 2002).

In Pakistan self-medication has mostly been studied in closed settings. Like according to one research $76 \%$ of university students in Karachi practice self-medication and the most common factor that encourages this behavior is previous experience with similar symptoms. (Zafar, Syed, Waqar, Zubairi \& Vaqar, 2008).

However, the effect of demographics on a person's self-medication practices has been a relatively understudied aspect. Furthermore, on most occasions, self-medication has been studied among university students only, so it would be meaningful to study this behavior among the general population. Therefore this study is aimed at determining the relationship of self-medication and demographic characteristics in the local settings of Karachi.

\subsection{Operational Definition}

Self-Medication: The use of medicines without medical supervision to treat one's illness.

OTC (Over-the-Counter) medicines: Medicines which an individual can get without a prescription.

Minor illness: Illness that doesn't prevent an individual from performing routine activities.

Prior experience: The previous encounter of similar illness and its remediation through a specific drug.

Recommendation: Recommendation of drugs from uncertified medical consultant including family, friends, pharmacists (salesperson at a drug store).

Convenience: Avoidance of difficulty or unnecessary efforts.

\subsection{Research Questions}

After the extensive review of previous literature on the subject matter, the four demographic characteristics i.e. gender, age, education and monthly income has been taken in the study to be tested against selfmedication. The aim is to examine that whether the gender, age, education and monthly income create the perception of self-medication or not. Therefore the research questions are formulated as follows.

- What is the statistical relationship between self-medication and gender?

$\circ$ What is the statistical relationship between self-medication and age?

- What is the statistical relationship between self-medication and education?

- What is the statistical relationship between self-medication and monthly income?

The demographic characteristics that have been taken in the study play a key role in a population. Thus examining their relationship with self-medication would be critical in having a deeper understanding of the subject matter.

\section{Review Of Literature}

Self-medication practices were not very common until the mid 90's. According to one survey, only $32 \%$ of the elderly patient aged 65 or above admitted to the hospital were reported to self-medicate before admission. And they had very little knowledge about the harm some products can cause (Batty, Oborne, Swift \& Jackson, 1997). A few years later in 2002 Shankar, Partha \& Shenoy conducted another study in Nepal to determine the prevalence and pattern of self-medication. In their study, they stated that self-medication practices are very common in developing countries and that the common reasons for this are non-availability of health personnel, while other reasons were mild illness and prior experience of treating the same illness.

Further, in 2007 two surveys were conducted to find out the practice of self-medication with antibiotics and/or antimalarial. Awad and Eltayeb conducted a research in Khartoum, Sudan to determine the self-medication behavior of university students. They reported that $79 \%$ of the sample population had used antibiotics or antimalarial without prescription. The most common reason indicated for self-medication was prior experience with the similar ailment. Later on, in the same year, another research was conducted to find out the prevalence and factors that lead to self-medication with antibiotics. It was concluded that $39.5 \%$ of the surveyed people had been using antibiotics without doctor's prescription and participants mainly relied on their prior experience and pharmacist advice for self-medication (Al-Azzam, Al-Husein, Alzoubi, \& Masadeh, 2007).

Next year in 2008 Albarrán \& Zapata carried out a survey in Chile to check the frequency and main reasons of self-medication among the households of Southern Chile. They stated that the frequency of selfmedication had reached $75 \%$ from $45 \%$ over the years from 1997 to 2008 .

The most frequent reasons were a minor illness, a subjective feeling of being able to manage one's pathology, convenience and lack of time. Although there are not many studies conducted in Pakistan on selfmedication practices, in 2008 students of Agha Khan University conducted a survey in 2 medical and 2 nonmedical universities of Karachi to study the prevalence, leading factors and knowledge of self-medication among university students. The prevalence was found to be $76 \%$ of the people surveyed with prior experience being the main reason for self-medication (Zafar, Syed, Waqar, Zubairi \& Vaqar, 2008).

Internationally, self-medication had been reported as being on the rise. So, to examine the use of branded medicines by the students of north India and the reason behind self-medication, a study was conducted that 
revealed that the major reasons behind self-medication were time-saving and not much need for advice for minor illness. Most of the respondents had a positive attitude towards self-medication for minor illness (Verma, Mohan, Pandey, 2010). In 2011 a descriptive cross-sectional study was conducted on 307 health sciences students in ACMU, Ethiopia. It states that $43.24 \%$ of surveyed population practice self-medication even though the majority of them had a negative attitude towards self-medication. The major reasons reported in this study were prior experience \& mildness of the illness (Gutema, Gadisa, Kidanemariam, Berhe, Berhe, Hadera \& Dagne, 2011).

Later in 2012, a study was conducted on 830 students enrolled in health or non-healthcare program of the city of Rio, Brazil to identify the prevalence and reasons associated with self-medication. The findings of the study state that $86.4 \%$ of the surveyed people self-medicate and no significant difference between healthcare and nonhealthcare students were observed regarding self-medication. And the factors influencing the behavior were gender, a number of children and medicine knowledge (da Silva, Soares \& Muccillo-Baisch, 2012). In the same year, another study was conducted in Nigeria that revealed that the self-medication practice is influenced by age, gender, and level of study (Osemene \& Lamikanra, 2012).

Recently, self-medication with antibiotics (SMA) has been reported among university students in many countries. So an anonymous online survey was conducted with the students of Shantou University (STU), China. The findings of the survey show that $47.8 \%$ of the respondents self-treated themselves with antibiotics. Older age, prior knowledge of antibiotics and higher monthly allowance were identified as independent risk factors for SMA (Pan, Cui, Zhang, Farrar, Law3 \& Ba-Thein, 2012).

\section{Research Methodology}

Pakistan Pharmaceutical Manufacturer Association (PPMA): PPMA visited to have in-depth information about different OTC and other therapeutic categories practices and policies and their enforcement in different pharmaceuticals strategic objectives. A quantitative study has been conducted in the local settings of Karachi to investigate demographics characteristics create the perception of self-medication. The nature of the study is crosssectional analysis. As cross-sectional data (data gathered from a population at a particular instant of time) has been used to examine the statistical relationship of demographics variables such as gender, age, education, monthly income and self-medication.

In order to test below described hypotheses, information was collected from different residential areas of different districts of Karachi. The detail of districts of Karachi given below:

Table 1: Disricts of Karachi

\begin{tabular}{|l|l|}
\hline 1. & Karachi Central \\
\hline 2. & District Malir \\
\hline 3. & District East \\
\hline 4. & District West \\
\hline 5. & District South \\
\hline
\end{tabular}

Source: internet

For the purpose of this study, following methodology is proposed.

\subsection{Hypotheses}

Hypotheses are specific testable guesses. They state that what you think will happen in a particular situation or circumstance. It consists of an independent and dependent variables and is derived on the basis of research or observation.

Based on our observations and the articles that we have reviewed, we have drawn the following hypotheses.

H1: Ho: Gender does not create the perception of self-medication.

HA: Gender does create the perception of self-medication.

H2: Ho: Age does not create the perception of self-medication.

HA: Age does create the perception of self-medication.

H3: Ho: Education does not create the perception of self-medication. HA: Education does create the perception of self-medication.

H4: Ho: Monthly income does not create the perception of self-medication. HA: Monthly income does create the perception of self-medication.

\subsection{Sampling Design}

Stratified random sampling and convenience sampling was used in Karachi to select number of individual respondents as the population is heterogeneous and was classified among sub-groups according to geographical locations. 


\subsection{Instrumentation:}

In order to collect data from above mentioned sources, interview and questionnaires was used. It is decided that a comprehensive questionnaires was developed containing a mix of open and close ended questions highlighting points regarding "self medications". Questionnaires was presented to the respondent of different geographic residential in different districts of Karachi.

Data is collected through self-administered questionnaire from different areas of Karachi. The questionnaire consists of 12 items in total. 7 of these items are taken from a research study on self-medication (Pan, Cui, Zhang, Farrar, Law3 \& Ba-Thein, 2012). And the other 3 items are taken from the beliefs about medications (Horne, Weinman \& Hankins, 1999).

\section{Frame Work of the Study}

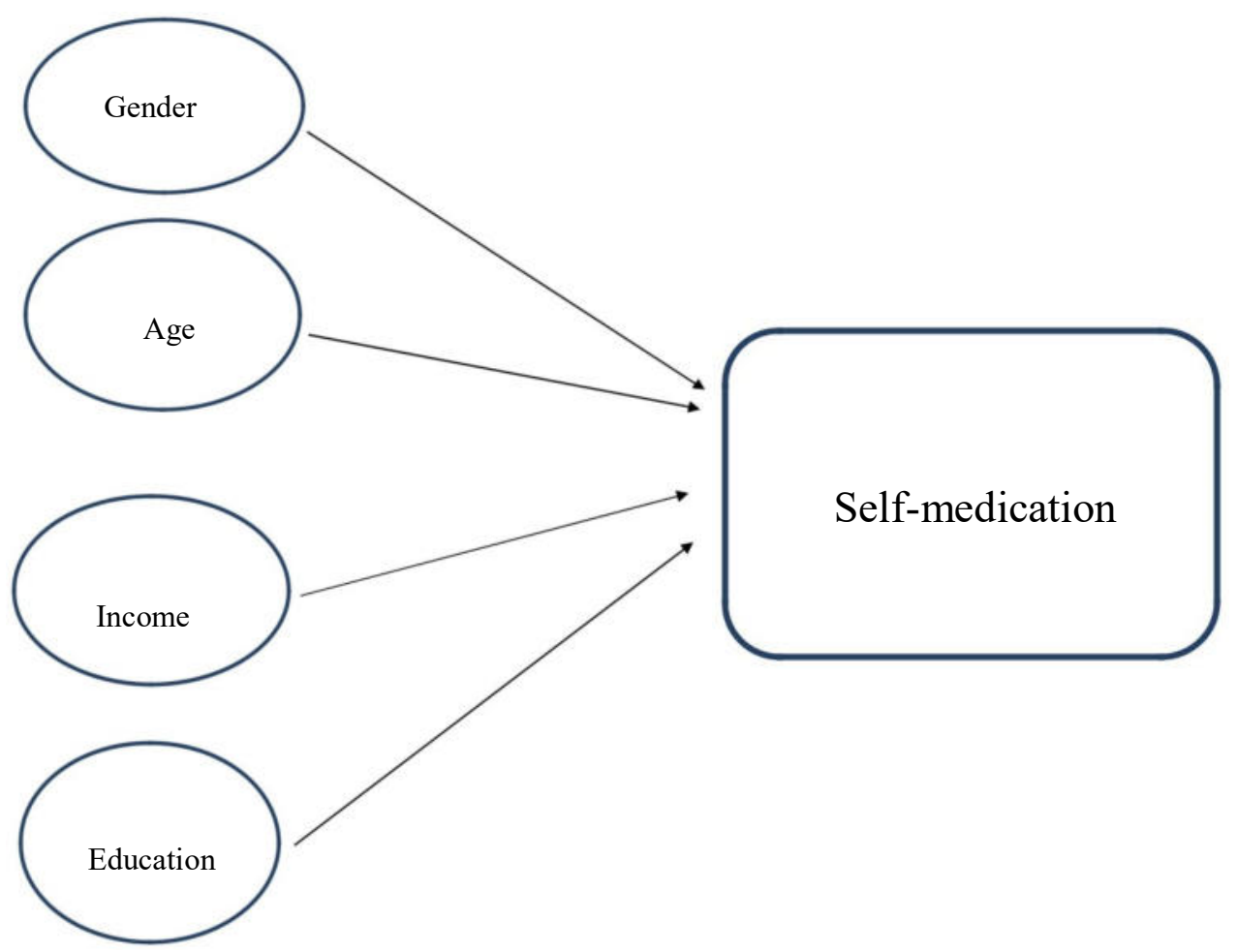

Our study is based on the above frame work. After extensive and thorough review of many articles on this subject matter, we have decided to examine the statistical relationship of self-medication with demographic variables of a population. Therefore we have taken four demographic characteristics (gender, age, education and monthly income) in our study model to be tested against self-medication.

\subsection{Data Analysis}

Keeping in view the nature of study, SPSS.20 was used for data analysis. This software help in data management from above mentioned sources, and statistical analysis of the data. Chi-square and Phi and Cramer's V test was used to measure attitude of consumer towards self medications. Descriptive and inferential statistics was used for showing interview and questionnaire results.

\subsection{Limitations}

Despite the fact that the study was quite effective in measuring the effects of critical variables on the subject matter, it had some fundamental limitations of a survey research.

The response of the participants could potentially be biased or exaggerated and might as well be inaccurate as respondents may not have been able to correctly recall past events.

Furthermore, as the research was conducted in Karachi, the use of English in survey questionnaire may have led people to misinterpret the questions. And thus affect the findings of the study. 
Selection of sample was based on convenience so it could be argued that it may not accurately represent the whole population and that conclusions may not be generalizable.

Finally, sample size and scope of research were limited due to time constraint which could have caused deviation in results.

\section{Research Findings}

The data is gathered from different areas of Karachi. During six months from July to December 2018 of data collection process, several people were asked for their participation, and few of them agreed to. The data was collected from a diverse pool of participants having different demographic characteristics like gender, age, education and monthly income as illustrated in Table 2.

\section{Figure 1}

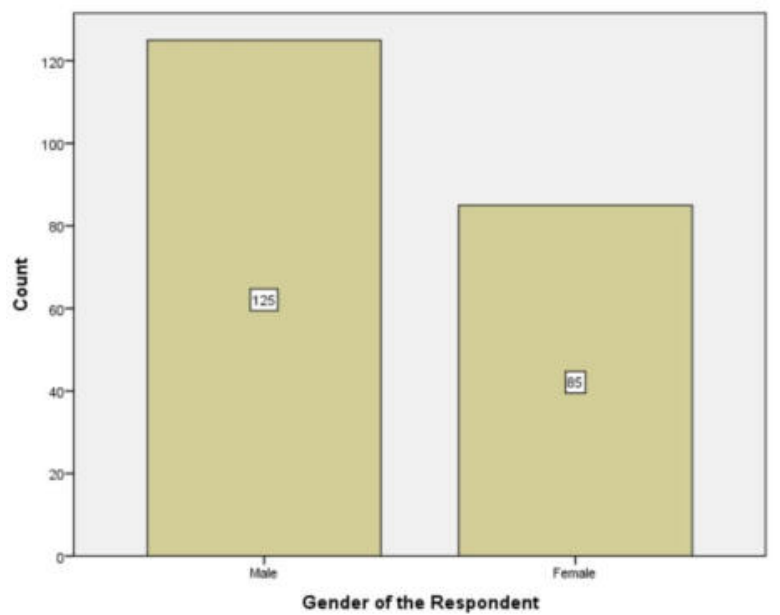

The percentage of male respondents $(59.52 \%)$ is much higher than female $(40.48 \%)$. This variation in percentage is much understandable as males are more likely to go to pharmacies to buy medicines and are relatively easy to approach.

Figure 2

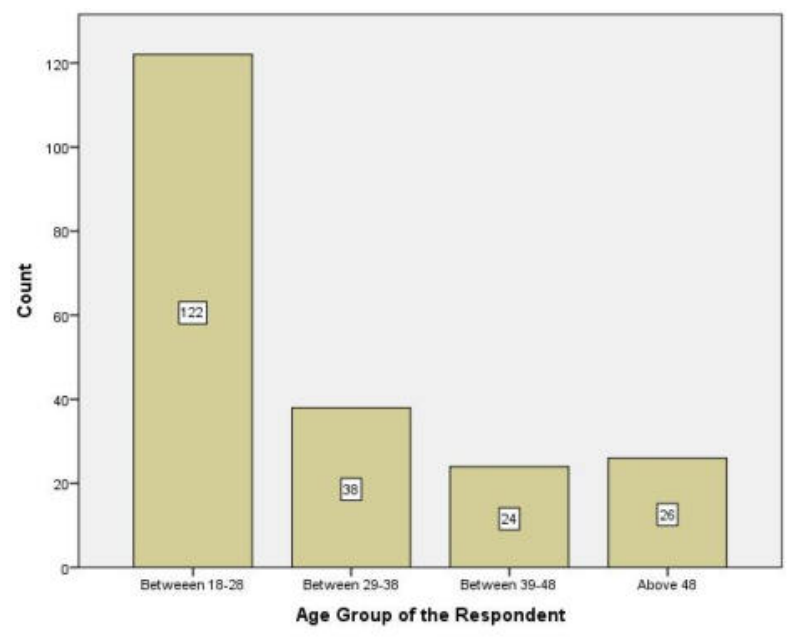

For accuracy of data, only adults with the age of 18 years and above were surveyed. Participants have been divided into four groups by their age. The percentage of respondents between ages 18 to 28 is $58.10 \%$, which is much higher than other age groups. The percentages of people of other age groups are; between 29 to $3818.10 \%$, between 39 to $4811.43 \%$ and above $4812.36 \%$. 
Figure 3

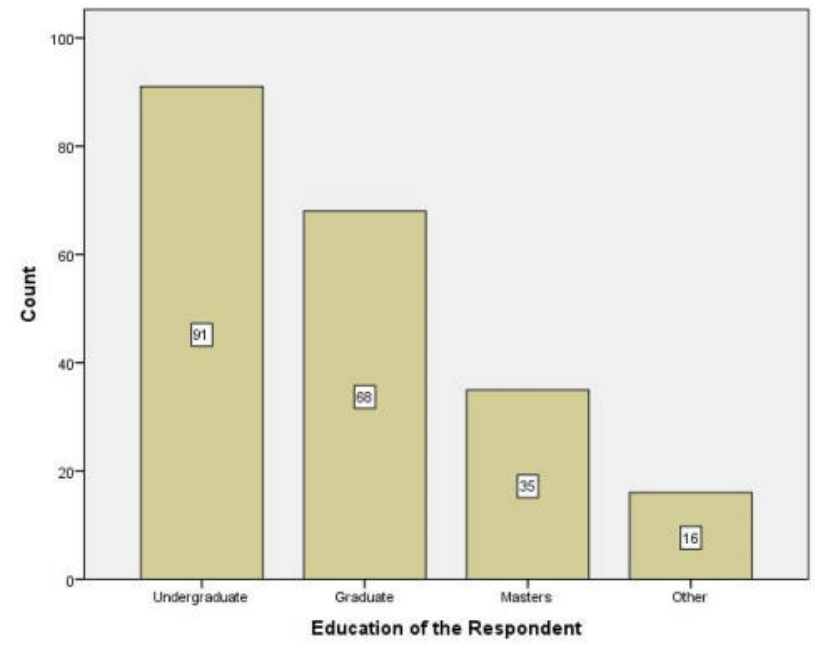

Education is another demographic variable that has been used to categorize the respondents. The percentage of undergraduate people $43.33 \%$ is higher than the other groups. The percentages of people of other groups are; graduate $32.38 \%$, Masters $16.67 \%$ and other $7.62 \%$.

\section{Figure 4}

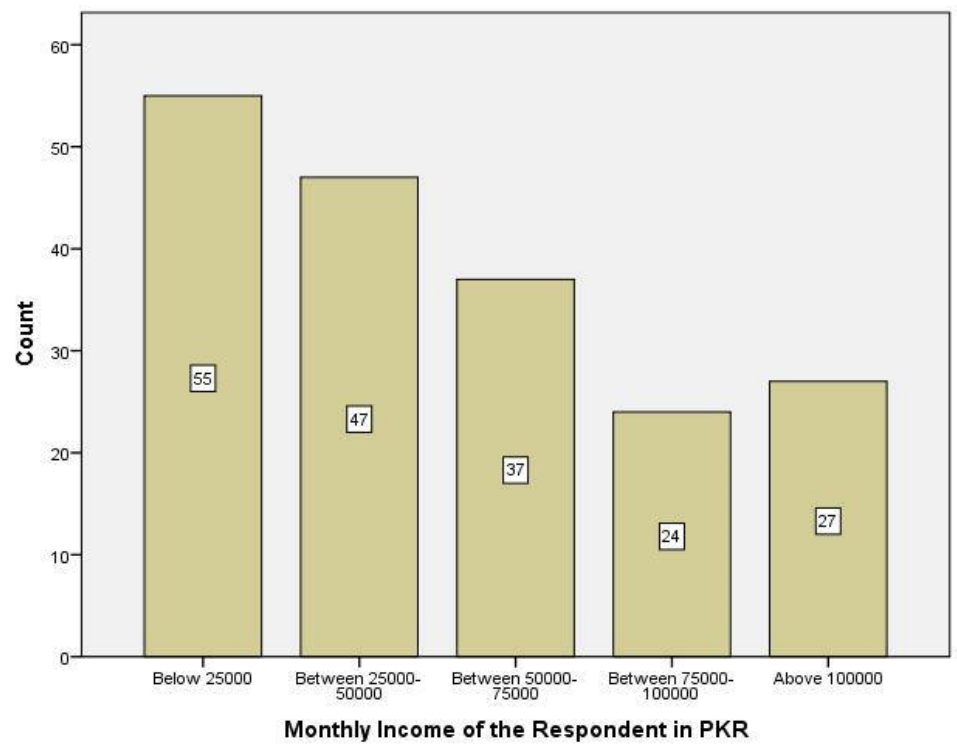

The final characteristic that has been used to categorize the data pool is monthly income (PKR). The respondents are very much equally distributed over the different income groups i.e. below 25,000 (28.95\%), between 25,000 to $50,000(24.74 \%)$, between 50,000 to $75,000(19.47 \%)$, between 75,000 to $1,00,000(12.63 \%)$ and above $1,00,000(14.21 \%)$.

As illustrated in Table the mean age of the respondent was between 28 and 38 years. While the average monthly income was between 50,000 and 75,000 (PKR). Moreover, the majority of the respondent belonged to the age group 18 to 28 years and were undergraduate. Finally the monthly income of most respondents was below 25,000 (PKR). 


15t

Table 2

\section{Respondent's Profile}

\section{Characteristic}

Male

Female

Between 18 to 28

Between 29 to 38

Between 38 to 48

Above 48

Graduate

Undergraduate

Masters

Other
Frequency

125

85

122

38

24

26

12.38

Education

Education

Below 25000
Monthly Income $\quad$ Between 25000 to 50000
Between 50000 to 75000
Betwen 75000 to 100000

Above 100000
91

68

35

16

16.67

7.62

14.21

\section{Percentage}

59.52

40.48

\subsection{Analysis of Pearson Chi Square}

The objective of the research study is to determine the statistical relationship between self-medication and demographics characteristics (gender, age, education and monthly income). For this purpose, Pearson chisquare test for independence has been used. 
The calculated chi-square values of demographic variables with self-medication are; gender and selfmedication 2.559, age and self-medication 1.641, education and self-medication $3.869 \&$ monthly income and self-medication 2.875 as shown in Table 3.

The tests for independence showed no significance which means that the calculated chi-square value of all demographic variables with self-medication is less than the tabulated value of chi-square at $\alpha=0.05$. Thus the null hypotheses cannot be rejected.

Table 3

Pearson Chi-Square

\begin{tabular}{|c|c|c|c|c|c|}
\hline \multicolumn{6}{|c|}{ Self-Medication } \\
\hline & & Value & Df & $\begin{array}{c}\text { Asymp.Sig } \\
\text { (2-sided) }\end{array}$ & Result \\
\hline \multirow[t]{2}{*}{ Gender } & Chi calculated & 2.559 & 1 & .110 & $\begin{array}{c}\text { Cannot reject } \\
\text { Ho. }\end{array}$ \\
\hline & Chi Tabulated & 3.841 & & & \\
\hline \multirow[t]{2}{*}{ Age } & Chi calculated & 1.641 & 3 & .650 & $\begin{array}{c}\text { Cannot reject } \\
\text { Ho. }\end{array}$ \\
\hline & Chi Tabulated & 7.815 & & & \\
\hline \multirow[t]{2}{*}{ Education } & Chi calculated & 3.869 & 3 & .276 & $\begin{array}{c}\text { Cannot reject } \\
\text { Ho. }\end{array}$ \\
\hline & Chi Tabulated & 7.815 & & & \\
\hline \multirow[t]{2}{*}{$\begin{array}{l}\text { Monthly } \\
\text { Income }\end{array}$} & Chi calculated & 2.875 & 4 & .579 & $\begin{array}{c}\text { Cannot reject } \\
\text { Ho. }\end{array}$ \\
\hline & Chi Tabulated & 9.488 & & & \\
\hline
\end{tabular}

Therefore, the test for independence suggested that the demographics characteristics i.e. gender, age, education and monthly income do not create the perception of self-medication among people and people self-medicate due to reasons other than these demographics.

Finally, since the test for independence has been non-significant for all demographic variables, therefore, Phi and Cramer's test wasn't performed to test the association.

\subsection{Hypotheses Summary}

\section{Hypothesis 1:}

Ho: Gender does not create the perception of self-medication. HA: Gender does create the perception of self-medication.

The test for independence shows no significance means there is no relationship between gender and selfmedication.

\section{Hypothesis 2:}

Ho: Age does not create the perception of self-medication.

HA: Age does create the perception of self-medication.

The chi-square test for independence shows no significance means there is no relationship between age and selfmedication.

\section{Hypothesis 3:}

Ho: Education does not create the perception of self-medication. HA: Education does create the perception of self-medication. 
The test for independence has been non-significant means that there is no relationship between education and selfmedication.

\section{Hypothesis 4:}

Ho: Monthly income does not create the perception of self-medication.

HA: Monthly income does create the perception of self-medication.

The test for independence has been non-significant means that there is no relationship between monthly income and self-medication.

\subsection{Key Findings}

The study confirms that the people behavior related to self-medication is not influenced by their demographic characteristics i.e. gender, age, education and monthly income, so that the demographic variables do not create the perception of self-medication.

\section{Conclusion}

The study showed that out of 210 respondents about $82 \%$ of the people practice self-medication. Of which $94.83 \%$ stated that they have practice self-medication for minor illness only while the other $5.17 \%$ of the respondents has tried it for major illness as well. And out of these $82 \%$ respondents who have practice self-medication, only $12 \%$ has experienced side-effects due to self-medication, upon which most of them consulted the doctor or stopped taking medicine. The major reasons stated by the respondents for engaging in self-medication are their prior experience, convenience, and recommendation of family/ friends/ others. Even though the majority of the respondents self-medicate but very few of them regarded it as a good practice. Most of the individual considered self-medication as only an acceptable behavior and a short term cure to the illness.

The behavior of $82 \%$ of the respondents (who practice self-medication) shows no evidence of being influenced by demographic characteristics.

Whereas, the $18 \%$ of the surveyed individuals, who do not practice self-medication hold a negative attitude towards self-medication and regard it as an unacceptable practice. They consider self-medication as a short-term cure for illness and are concerned about the long-term effects of medicines.

\section{$>$ References:-}

World Health Organization. (2000). Guidelines for the regulatory assessment of Medicinal Products for use in self-medication

Dukes MN: drug utilization studies, copenhagen: WHO regional publications; 1993

Wajngarten M: editorial. Rev Assoc Med Bras 2001, 47(4): 269-295

Chang, F. R., \& K Trivedi, P. (2003). Economics of self-medication: theory and evidence. Health economics, 12(9), 721-739.

Hussain, A., \& Khanum, A. (2008). Self medication among university students of Islamabad, Pakistan-a preliminary study. Southern Med Review, 1(1), 14-16.

Shankar, P. R., Partha, P., \& Shenoy, N. (2002). Self-medication and non-doctor prescription practices in Pokhara valley, Western Nepal: a questionnaire-based study. BMC family practice, 3(1), 17.

Zafar, S. N., Syed, R., Waqar, S., Zubairi, A. J., Vaqar, T., Shaikh, M \& Saleem, S. (2008). Self-medication amongst university students of Karachi: prevalence, knowledge and attitudes. Journal of the Pakistan Medical Association, 58(4), 214.

Batty, G. M., Oborne, C. A., Swift, C. G., \& Jackson, S. H. D. (1997). The use of over-the-counter medication by elderly medical in-patients. Postgraduate medical journal, 73(865), 720-722.

Shankar, P. R., Partha, P., \& Shenoy, N. (2002). Self-medication and non-doctor prescription practices in Pokhara valley, Western Nepal: a questionnaire-based study. BMC family practice, 3(1), 17.

Awad, A. I., \& Eltayeb, I. B. (2007). Self-medication practices with antibiotics and anti malarials among Sudanese undergraduate university students. Annals of Pharmacotherapy, 41(7-8), 1249-1255. 
Al-Azzam, S., Al-Husein, B., Alzoubi, F., \& Masadeh, M. (2007). Self-medication with antibiotics in Jordanian population. International journal of occupational medicine and environmental health, 20(4), 373-380.

Albarrán, K. F., \& Zapata, L. V. (2008). Analysis and quantification of self-medication patterns of customers in community pharmacies in southern Chile. Pharmacy world \& science, 30(6), 863-868.

Verma, R. K., Mohan, L., \& Pandey, M. (2010). Evaluation of self-medication among professional students in North India: proper statutory drug control must be implemented. Evaluation, 3(1), 60-64.

Gutema, G. B., Gadisa, D. A., Kidanemariam, Z. A., Berhe, D. F., Berhe, A. H., Hadera, M.

\& Dagne, A. W. (2011). Self-Medication Practices among Health Sciences Students: The Case of Mekelle University.

da Silva, M. G. C., Soares, M. C. F., \& Muccillo-Baisch, A. L. (2012). Self-medication in university students from the city of Rio Grande, Brazil. BMC public health, 12(1), 339.

Osemene, K. P., \& Lamikanra, A. (2012). A study of the prevalence of self-medication practice among university students in Southwestern Nigeria. Tropical Journal of Pharmaceutical Research, 11(4), 683-689.

Pan, H., Cui, B., Zhang, D., Farrar, J., Law, F., \& Ba-Thein, W. (2012). Prior knowledge, older age, and higher allowance are risk factors for self-medication with antibiotics among university students in southern China. PloS one, 7(7), e41314.

Horne, R., Weinman, J., \& Hankins, M. (1999). The beliefs about medicines questionnaire: the development and evaluation of a new method for assessing the cognitive representation of medication. Psychology and health, 14(1), 1-24.

Comprehensive Statistics Concepts \& Methods Syed Khursheed Alam Iqra Publishers. 